Polymer Journal, Vol. 3, No. 5, pp 538-550 (197 $\left.2^{2}\right)$

\title{
Solution Properties of Synthetic Polypeptides. XI. Solvent Effect on Helix-Coil Transition in Polypeptides
}

\author{
Norio Sayama, Kenji Kida, Takashi Norisuye, Akio Teramoto, ${ }^{*}$ \\ and Hiroshi Fujrta \\ Department of Polymer Science, Osaka University, Toyonaka, Japan.
}

(Received November 15, 1971)

\begin{abstract}
A number of synthetic polypeptides are known to undergo "inverse" thermal helix - coil transitions in solvent mixtures containing an "active" solvent such as dichloroacetic acid (DCA). A statistical mechanical theory of the effects of the active component on the transition is developed here on the assumption that the active solvent exists in the form of a dimer and that the dimerized molecules react with pairs of $\mathrm{CO}$ and $\mathrm{NH}$ groups of randomly coiled peptide units. Expressions are derived for the equilibrium constant, $u$, of helix formation and the cooperativity parameter, $\sigma$, and they are used to analyze data obtained for $\operatorname{poly}(\gamma$-benzyl-L-glutamate) and poly( $\beta$-benzyl-Laspartate) in mixtures of DCA and ethylene dichloride as functions of temperature and solvent composition. The values of the transition parameters so determined make it possible to understand not only why and how the observed values of transition enthalphy $\Delta H$ and cooperativity parameter $\sigma$ depend on temperature and solvent composition, but also the general features of inverse transitions in quantitative terms. An important conclusion is that $\sigma$ is profoundly affected by the binding of the active solvent on the polypeptide chain.
\end{abstract}

KEY WORDS Helix-Coil Transition / Polypeptide / Transition Enthalpy / Cooperativity Parameter / Solvent Effect / Poly $(\gamma$-benzylL-glutamate) / Poly( $\beta$-benzyl-L-aspartate) /

It is well known that for synthetic polypeptides in dilute solution, many of them undergo thermally induced helix-coil transition of the "inverse" type when the solvent is a binary mixture consisting of a non- or slightly-polar helix-supporting solvent and a strong organic acid. $^{1,2}$ The inversion of the direction of transition is generally attributed to the binding of the acid with $\mathrm{CO}$ and $\mathrm{NH}$ groups of peptide units. This effect of the "active" solvent component was first formulated theoretically by Schellman, ${ }^{3}$ and then put into a more elaborated consideration by several authors. ${ }^{4-6}$ The basic assumption of these authors is that the active solvent interacts independently with either $\mathrm{CO}$ or NH group of each peptide unit. However, it does not appear to fit the actual situation in the system where a carboxylic acid such as dichloroacetic acid (DCA) is used as the active solvent, because such an acid in a liquid of

* To whom correspondence should be addressed. low dielectric constant is known to exist only in associated forms, predominantly as a dimer, ${ }^{7}$ so that it can not interact independently with each active site of the peptide unit. If this is the case, the theoretical formulations of the previous authors ${ }^{3-6}$ will have to be modified.

In this paper, such a modification is presented, assuming that the active solvent exists as a dimer in an "inert" solvent and that the dimerized active solvent hydrogen bonds with a pair of $\mathrm{CO}$ and $\mathrm{NH}$ groups of each peptide unit. Here, "inert solvent" is taken to mean a liquid which essentially has no interaction with these active sites of peptide residues. The derived theoretical expressions are applied to detailed experimental data obtained with poly $(\gamma$-benzyl-Lglutamate)(PBLG) and poly( $\beta$-benzyl-L-aspartate) (PBLA) in mixtures of DCA and ethylene dichloride (EDC), with the basic premise that these organic solvents satisfy the assumptions underlying the theory developed. A general treatment of the solvent effect on the helix-coil 
transition in polypeptide is presented as a supplementary material in the Appendix.

\section{THEORETICAL}

\section{Formulation of Solvent Effects}

A nonionic homopolypeptide dissolved in a mixture of an active solvent and an inert solvent is considered. At a given temperature, the polypeptide molecule may be in the state of interrupted helix. For simplicity, a peptide unit contained in a helical section is called a helical unit, and that contained in a randomly coiled section is called a coiled unit. It is assumed that the interaction of each solvent component with side chains of the polypeptide is not affected by the conformational state of the macromolecule.

It is assumed that the active solvent exists in the form of dimer-for DCA it is known that its dimerized form has the structure: ${ }^{7}$

$$
\mathrm{HCl}_{2} \mathrm{C}-\mathrm{C}_{\mathrm{O}-\mathrm{H} \cdots \mathrm{O}}^{\mathrm{O} \cdots \mathrm{H}-\mathrm{O}} \mathrm{C}_{\mathrm{C}-\mathrm{CCl}_{2} \mathrm{H}}
$$

where three dots represent a hydrogen bond. No convincing information is available about what happens when this DCA dimer comes close to a peptide unit. It is assumed here that the following reaction takes place between these two species:

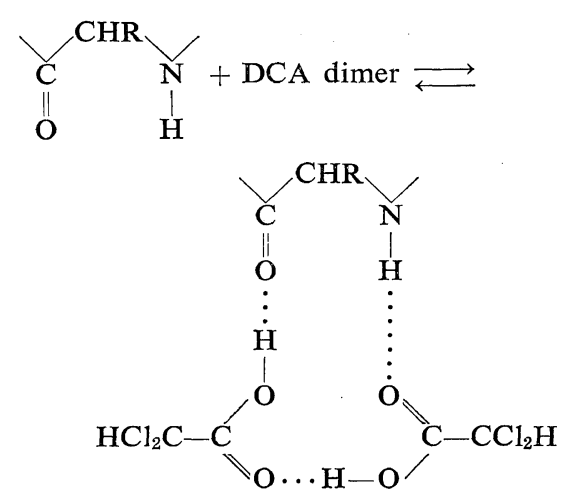

which is acceptable from a stereochemical point of view. It is seen that, in this reaction, one hydrogen bond is lost from a DCA dimer and two hydrogen bonds are produced between the dimer and a pair of "free" CO and $\mathrm{NH}$ groups of each peptide unit. Thus the proposed reaction lowers the ethalpy of the system, i.e., stabilizes the system enthalpically. On the other hand, if the DCA dimer reacted independently with each of $\mathrm{CO}$ and $\mathrm{NH}$ groups, no appreciable change in enthalpy would occur, because the number of hydrogen bonds remains unchanged before and after the reaction.

The statistical weight of a helical unit inside a helical section of the polypeptide in the given mixed solvent is denoted by $u$. Following the procedure described by Bixon and Lifson, ${ }^{6}$ it can be shown that this quantity is expressed by

$$
u=u_{0} /(1+K a)
$$

where $u_{0}$ is the value of $u$ in the system containing no active solvent, $K$ is the equilibrium constant for the proposed reaction between dimerized active solvent and peptide unit, and $a$ is the thermodynamic activity of the dimerized active solvent in the mixture.

In statistical mechanical treatments of helixcoil transitions in polypeptides, the peptide unit at either end of any helical section must be distinguished from other units inside the same helical section. Its statistical weight relative to that of the the coiled unit in the absence of the active solvent is denoted by $\sqrt{\sigma_{0}}$. Then, according to Nagai's theory, ${ }^{8}$ it can be shown (Appendix) that the helical content and other conformational characteristics are expressed in terms of $u$ defined above and of $\sigma$ defined by

$$
\sqrt{\sigma}=\sqrt{\sigma_{0}} /(1+K a)^{2}
$$

The derivation of eq 1 and 2 is given in the Appendix.

These expressions for $u$ and $\sqrt{\sigma}$ refer to the particular reaction mechanism assumed above. Different expressions are to be obtained for other solvation mechanisms. Some of them are presented in the Appendix.

\section{Determination of Parameters $u$ and $\sigma$}

Based on the Nagai theory ${ }^{8}$ (essentially equivalent to the Zimm-Bragg theory ${ }^{9}$ ) for homopolypeptides in dilute solution, Okita, et al. ${ }^{10}$ have shown that the helical content, $f_{N}$, of a polypeptide having a degree of polymerization $N$ can be expressed, with sufficient accuracy, by

$$
f_{N}=f[1-2 \sqrt{f(1-f)} /(N \sqrt{\sigma})]
$$

provided $N \gg 1, \sqrt{\sigma} \ll 1$, and $N \sqrt{\sigma}>2$. Here 
$f$, the value of $f_{N}$ for $N=\infty$, is related to a combined parameter $z=(\ln u) / 2 \sqrt{\sigma}$ by the equaation

$$
f=(1 / 2)\left(1+z / \sqrt{1+z^{2}}\right)
$$

These equations indicate that a plot of $f_{N}$ at a fixed temperature against $N^{-1}$ gives a straight line and that one can evaluate $\ln u$ and $\sqrt{\sigma}$ from the intercept and slope of the line. For a polypeptide sample heterogeneous in $N$, one may replace $N$ in eq 3 by the number-average of polymerization, $\bar{N}_{n}$.

Repetition of the above-mentioned process at other temperatures may yield data for $\ln u$ as a function of temperature. The transition enthalpy at a fixed composition of the active solvent, $\Delta H$, can then be computed by using the relation:

$$
\Delta H=-R \partial(\ln u) / \partial(1 / T)
$$

Substitution of eq 1 into eq 5 yields

$$
\Delta H=\Delta H_{0}-\frac{K a}{1+K a}\left(\Delta H_{\mathrm{a}}-\Delta H_{\mathrm{m}}\right)
$$

where

$$
\begin{aligned}
& \Delta H_{0}=-R \partial\left(\ln u_{0}\right) /(1 / T) \\
& \Delta H_{\mathrm{a}}=-R \partial(\ln K) / \partial(1 / T) \\
& \Delta H_{\mathrm{m}}=R \partial(\ln a) / \partial(1 / T)
\end{aligned}
$$

Here $\Delta H_{0}$ is the change in enthalpy accompanying the conformational transition of the polypeptide chain from random coil to helix in the inert solvent, $\Delta H_{\mathrm{a}}$ is the enthalpy for the binding of a dimer of the active solvent with a pair $\mathrm{CO}$ and $\mathrm{NH}$ groups, and $\Delta H_{\mathrm{m}}$ is the partial molar enthalpy of the dimerized active solvent in the mixture considered. Both $a$ and $\Delta H_{\mathrm{m}}$ can be determined from vapor pressure measurements at various temperatures and for various compostions of the given solvent mixtures. Therefore, our problem amounts to developing a procedure which permits the evaluation of $\Delta H_{0}$, $\Delta H_{\mathrm{a}}$, and $K$ from experimental data for $u(T, a)$. It is noted that expressions analogous to eq 6 have been derived by Peller ${ }^{4}$ and Bixon and Lifson ${ }^{6}$ on the basis of different solvation mechanisms.

\section{Procedure for Data Analysis}

Method I. This method utilizes data for $u$ as a function of $T$ and $a$. If eq 1 holds, then it follows that values of $u^{-1}$ at a fixed temperature plotted against $a$ should give a straight line, and $u_{0}$ and $K$ at that temperature can be evaluated from the ordinate intercept and slope of the line. This operation is repeated at other temperatures to determine both $u_{0}$ and $K$ as functions of $T$. Substitution of the data thus obtained into eq 7 and 8 allows the computation of $\Delta H_{0}$ and $\Delta H_{\mathrm{a}}$.

Method II. This method resorts to data for the transition temperature $T_{\mathrm{c}}$ and $\Delta H\left(T_{\mathrm{c}}, a\right)$. It is more restrictive than Method I, because $\Delta H_{0}$, $\Delta H_{\mathrm{a}}$, and $\Delta H_{\mathrm{m}}$ must be assumed to be independent of temperature and, moreover, the coefficients $A$ and $B$ in the exppessions

$$
\begin{gathered}
u_{0}=A \exp \left(-\Delta H_{0} / R T\right), \\
K=B \exp \left(-\Delta H_{\mathrm{a}} / R T\right)
\end{gathered}
$$

must be treated as constant. In actual cases, however, these assumptions are obeyed quite accurately.

Since, by definition, $u=1$ at $T=T_{\mathrm{c}}$, it follows from eq 1 that

$$
A \exp \left(-\Delta H_{0} / R T_{\mathrm{c}}\right)=1+a B \exp \left(-\Delta H_{\mathrm{a}} / R T_{\mathrm{c}}\right)
$$

where eq 10 have been inserted. Writing eq 6 at $T=T_{\mathrm{c}}$, we obtain

$$
\Delta H_{\mathrm{c}}=\Delta H_{0}-\Delta H_{\mathrm{a}}{ }^{\prime}+\left(\Delta H_{\mathrm{a}}{ }^{\prime} / A\right) \exp \left(\Delta H_{0} / R T_{\mathrm{c}}\right)
$$

where

$$
\begin{aligned}
\Delta H_{\mathrm{c}} & =\Delta H \quad \text { at } \quad T=T_{\mathrm{c}} \\
\Delta H_{\mathrm{a}}{ }^{\prime} & =\Delta H_{\mathrm{a}}-\Delta H_{\mathrm{m}}
\end{aligned}
$$

One chooses a reference temperature $T_{*}$ in the central part of the temperature region in which $T_{\mathrm{c}}$ 's for chosen values of $a$ appear, and then determines $u_{0}$ corresponding to this temperature by linear extrapolation of the plot of $u^{-1} v s$. $a$ to $a=0$. Denoting this $u_{0}$ value by $\left(u_{0}\right)_{*}$, it follows from eq 10 and 12 that

$$
\begin{aligned}
\Delta H_{\mathrm{c}}= & \Delta H_{0}-\Delta H_{\mathrm{a}}{ }^{\prime}+\left[\Delta H_{a}{ }^{\prime} /\left(u_{0}\right)_{*}\right] \\
& \times \exp \left[\left(\Delta H_{0} / R\right)\left(1 / T_{\mathrm{c}}-1 / T_{*}\right)\right]
\end{aligned}
$$

If $\Delta H_{0} / R T_{*}$ is not too large in comparison with unity, a plot of $\Delta H_{\mathrm{c}} v \mathrm{~s} .\left(1 / T_{\mathrm{c}}-1 / T_{*}\right)$ will become linear over a fairly wide range of abscissa, and the ordinate intercept $P$ and the slope $Q$ at $T=T_{*}$ may be determined with precision. Then eq 15 and the values of $P, Q$, and $\left(u_{0}\right)_{*}$ allow 
$\Delta H_{0}$ and $\Delta H_{\mathrm{a}}{ }^{\prime}$ to be computed. Since $\Delta H_{\mathrm{m}}$ can be determined separately, the desired $\Delta H_{\mathrm{a}}$ can also be derived. Once $\Delta H_{0}$ and $\Delta H_{\mathrm{a}}$ are known, it is possible to compute $A$ and $B$ from eq 10 and 11. In this way, too, one can determine all the quantities associated with the present treatment of solvent effects on helix-coil transition.

\section{EXPERIMENTAL RESULTS}

Fractionated samples of PBLG and PBLA were used. Their typical characteristics can be seen in the previous papers of this series. ${ }^{11,12}$ Both DCA and EDC were used after careful fractional distillation. In the subsequent presentation, the compositions of their mixtures are indicated by their volume ratios at $25^{\circ} \mathrm{C}$.

Specific rotations at a fixed wavelength $\lambda$ $\left([\alpha]_{\lambda}\right)$ and mean residue rotations $\left(\left[m^{\prime}\right]_{\lambda}\right)$ were measured under a variety of solvent conditions as functions of molecular weight, and then converted to helical content $f_{N}$ by means of the relations: ${ }^{12,13}$

PBLG in DCA-EDC mixtures:

$$
f_{N}=\left(\left[m^{\prime}\right]_{436}+50.7\right) / 78.4
$$

PBLA in DCA-EDC mixtures:

$$
f_{N}=-\left([\alpha]_{546}+13\right) / 157
$$

Vapor pressures of DCA-EDC mixtures were measured with a glass cell of $30-\mathrm{m} l$ capacity equipped with a mercury manometer. The whole assembly was immersed in a water bath regulated to within $\pm 0.01^{\circ}$, and the pressures were determined to the accuracy of $0.01 \mathrm{mmHg}$ at a number of temperatures between 5 and $40^{\circ} \mathrm{C}$. The values obtained for pure EDC were in close agreement with literature data ${ }^{14}$ over the range of temperature examined.

\section{Features of the Transition Curves}

Figures 1 and 2 show typical transition curves for PBLG and PBLA in DCA-EDC mixtures. Both polypeptides in these solvent mixtures undergo a transition from coil to helix with raising temperature (i.e., an inverse transition). For either polypeptide, the transition curve shifts toward higher temperature and becomes less steep as the DCA content in the mixture is increased. Upon further increase in DCA con-

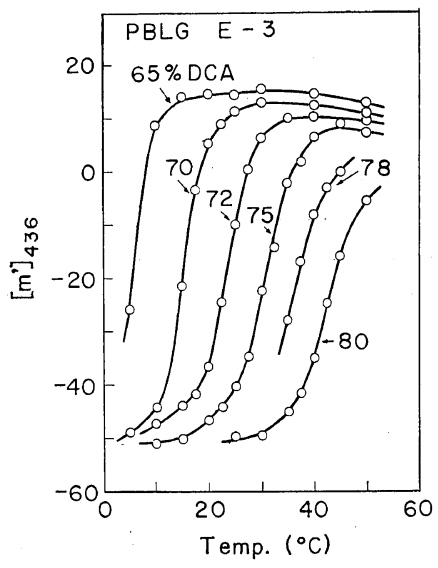

Figure 1. Temperature dependence of mean residue rotation at wavelength $436 \mathrm{~m} \mu,\left[\mathrm{m}^{\prime}\right]_{436}$, for a PBLG sample $\left(\bar{N}_{n}=584\right)$ in DCA-EDC mixtures. Composition of each DCA-EDC mixture is indicated by vol $\%$ of DCA at $25^{\circ} \mathrm{C}$.

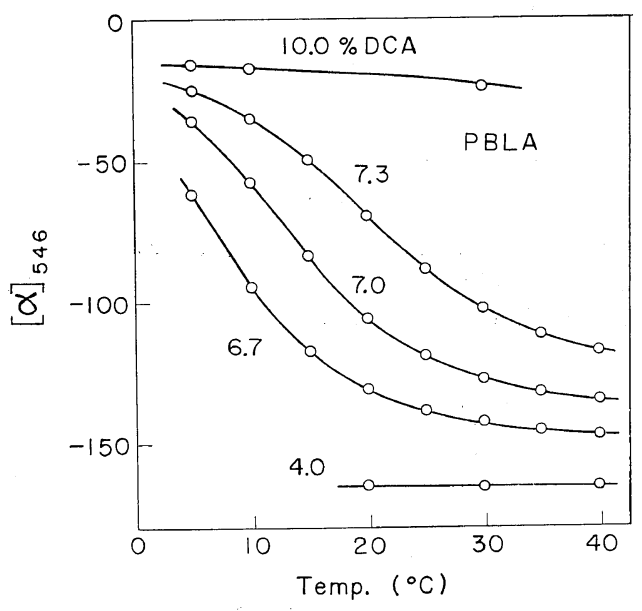

Figure 2. Temperature dependence of specific rotation at $546 \mathrm{~m} \mu,[\alpha]_{546}$, for a PBLA sample $\left(\bar{N}_{n}=\right.$ 1130) in DCA-EDC mixtures. Composition of each DCA-EDC mixture is indicated by vol\% of DCA at $25^{\circ} \mathrm{C}$.

tent, there appears a broad maximum. The helical content corresponding to this maximum is considerably lower than unity. A similar behavior can be observed in the data for PBLG and poly $(\gamma$-methyl-L-glutamate) (PMLG) in DCA -EDC mixtures by Nakajima and Hayashi. ${ }^{15}$ Since $[\alpha]_{\lambda}$ and $\left[m^{\prime}\right]_{\lambda}$ are increasing functions of helical content, these facts imply that the transi- 
tion of either PBLG or PMLG-EDC mixtures becomes normal above a certain temperature.

Figure 3 illustrates the dependence of the transition curve on molecular weight with the data from three PBLA samples in a DCA(6.7)$\operatorname{EDC}(93.3)$ mixture. Features of the data at other solvent compositions and also those for PBLG were entirely similar to the those depicted in Figure 3. Part of these data for PBLG has

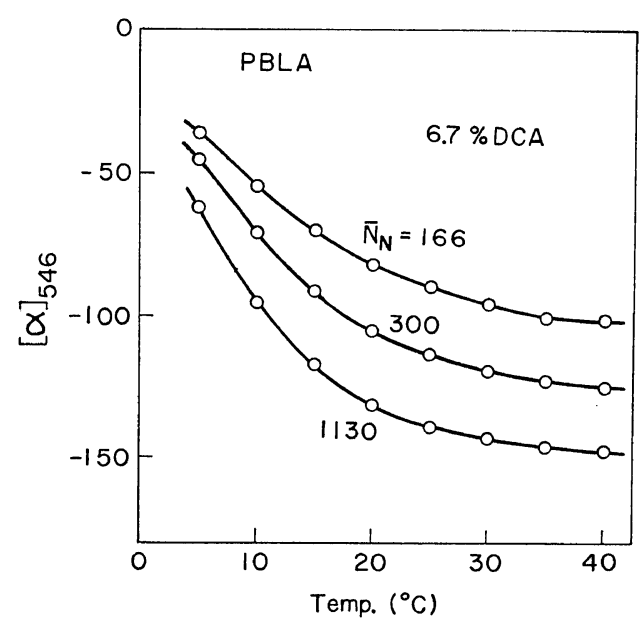

Figure 3. Temperature dependence of $[\alpha]_{546}$ for PBLA in a DCA-EDC mixture (6.7 vol\% of DCA) as a function of molecular weight.

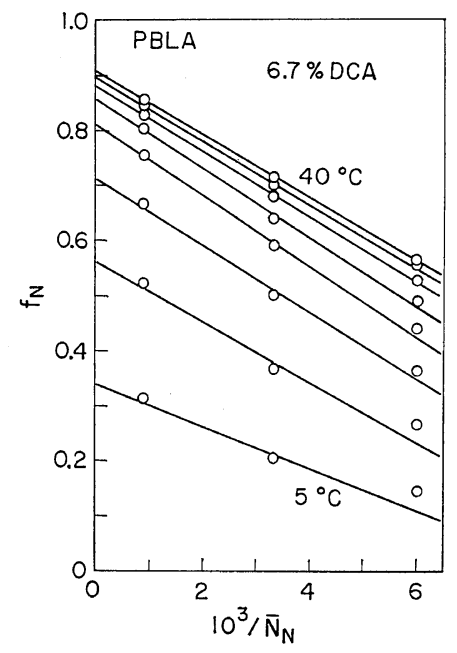

Figure 4. Plots of $f_{N}$ vs. $\bar{N}_{n}^{-1}$ for PBLA in a DCA-EDC mixture (6.7 vol\% of DCA) at 40,35 , $30,25,20,15,10$, and $5^{\circ} \mathrm{C}$ from top to bottom. already been presented in the authors' previous paper. $^{12}$

\section{Evaluation of Transition Parameters}

Figure 4 shows plots of $f_{N} v s . \bar{N}_{n}^{-1}$ constructed from the data given in Figure 3. Figures 5 and 6 show similar plots for PBLA in other DCAEDC mixtures. As expected from eq 3, the plotted points at each temperature follow a

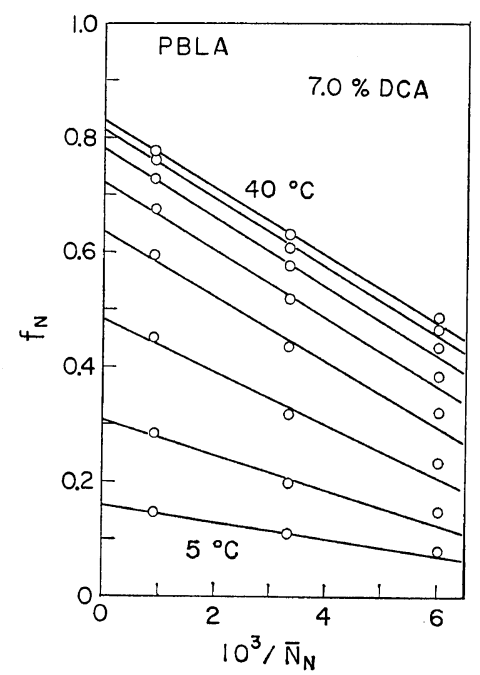

Figure 5. Plots of $f_{N}$ vs. $\bar{N}_{n}^{-1}$ for PBLA in a DCA-EDC mixture (7.0 vol\% of DCA) at the same temperatures as those in Figure 4.

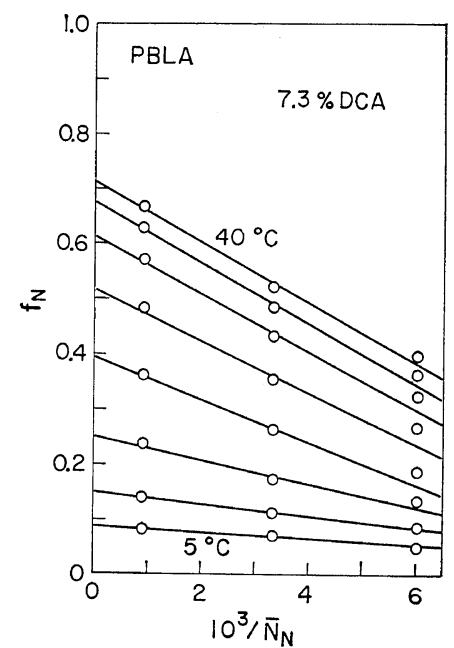

Figure 6. Plots of $f_{N}$ vs. $\bar{N}_{n}^{-1}$ for PBLA in a DCA-EDC mixture (7.3 vol\% of DCA) at the same temperatures as those in Figure 4. 
straight line. Results obtained for PBLG in DCA-EDC mixtures of various compositions were similar, and confirmed the authors' previous results reported elsewhere. ${ }^{12}$ From the straight lines obtained, $\sigma$ and $\ln u$ were calculated by the method outlined in the theoretical section. Since the values of $\sigma$ for each pair of polymer and solvent were approximately independent of temperature, only their averages are presented in Table $\mathrm{I}$. If one considers the uncertainty involved in the determination of $\sigma$, it may be

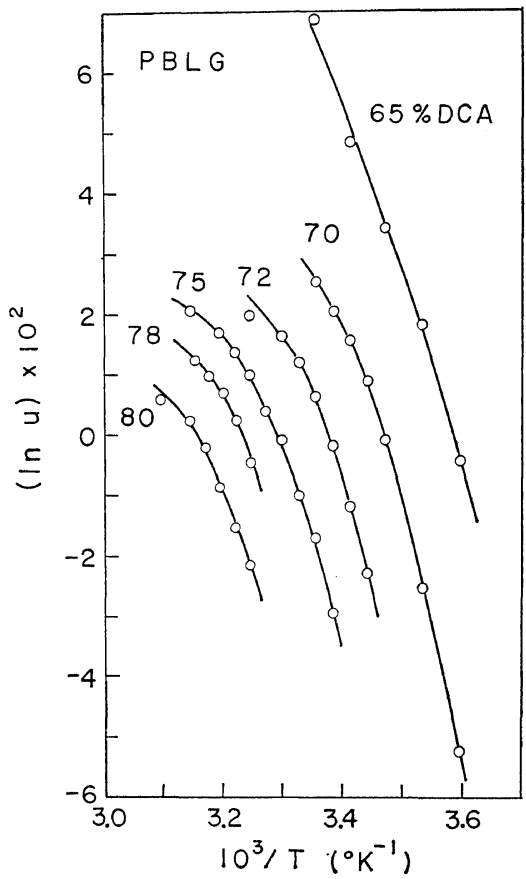

Figure 7. Plots of $\ln u v s .1 / T$ for PBLG in DCAEDC mixtures of the indicated DCA content. be concluded from this table that $\sigma$ is constant in the range of solvent composition studied.

Figure 7 shows plots of $\ln u v s .1 / T$ for PBLG in DCA-EDC mixtures of different compositions. The data points for each mixture follow a curve having downward curvature, indicating that $\Delta H$ decreases with increasing temperature. The corresponding data for PBLA in mixtures of DCA and EDC behave similarly, as shown in Figure 8. Table I lists the values of $\Delta H$ for these systems at their transition points $T_{\mathrm{c}}$. Comparison of these $\Delta H$ values with the data from calorimetric measurements must be made with caution, as will be discussed in a forthcoming paper of this series. ${ }^{16}$

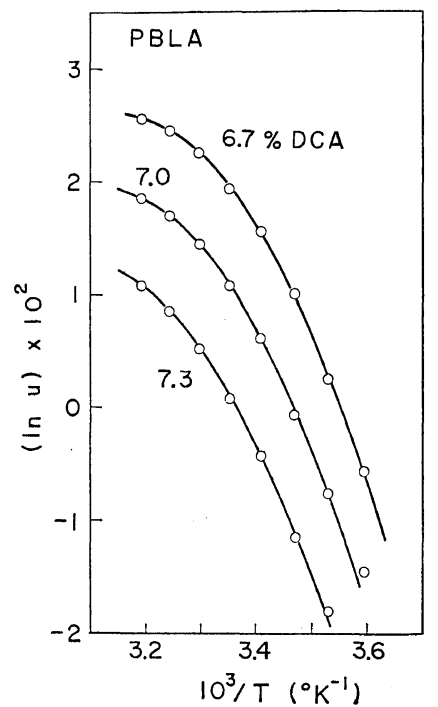

Figure 8. Plots of $\ln u v s .1 / T$ for PBLA in the three DCA-EDC mixtures.

Table I. Transition parameters for PBLG and PBLA in mixtures of DCA and EDC

\begin{tabular}{ccccc}
\hline Polypeptide & $\begin{array}{c}\text { Solvent composition, } \\
\text { vol\% of DCA }\end{array}$ & $\sqrt{\sigma} \times 10^{2}$ & $\begin{array}{c}\Delta H \text { at } T_{\mathrm{c}}, \\
\text { cal/mol }\end{array}$ & $T_{\mathrm{c}},{ }^{\circ} \mathrm{C}$ \\
\hline PBLA & 6.7 & $1.00 \pm 0.05$ & $270 \pm 20$ & 8.3 \\
& 7.0 & $1.05 \pm 0.05$ & $230 \pm 20$ & 15.0 \\
PBLG & 7.3 & $1.06 \pm 0.09$ & $175 \pm 15$ & 24.1 \\
& 65 & $1.0 \pm 0.3$ & $800 \pm 50$ & 5.1 \\
& 70 & $0.97 \pm 0.14$ & $780 \pm 50$ & 15.2 \\
& 72 & $0.93 \pm 0.10$ & $720 \pm 40$ & 23.5 \\
& 75 & $0.96 \pm 0.10$ & $600 \pm 50$ & 30.7 \\
& 78 & $0.89 \pm 0.10$ & $580 \pm 60$ & 36.3 \\
& 80 & $0.81 \pm 0.10$ & $500 \pm 50$ & 43.3 \\
\hline
\end{tabular}




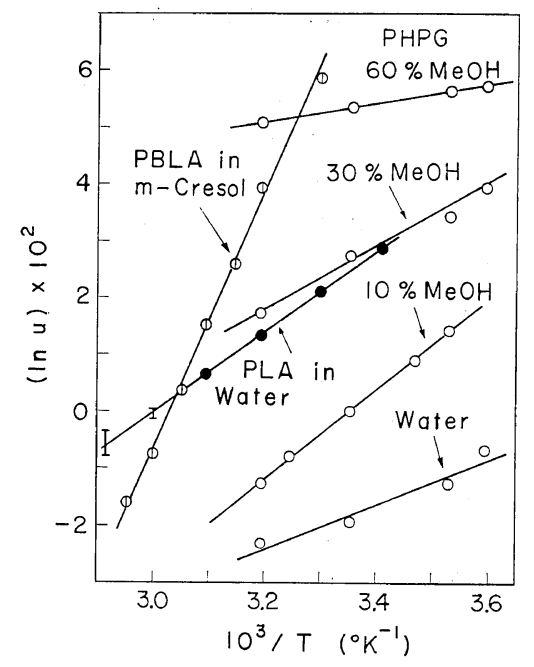

Figure 9. Plots of $\ln u v s .1 / T$ for various polypeptide-solvent systems in which normal transitions occur: (1), PBLA in $m$-cresol; $11 \bigcirc$, $\operatorname{poly}\left(N^{5}\right.$ (3-hydroxypropyl)-L-glutamine) (PHPG) in watermethanol mixtures (composition is indicated by wt $\%$ of methanol); 10 , poly(L-alanine) (PLA) in water. ${ }^{17}$

For comparison, plots of $\ln u v s$. $1 / T$ for several systems $^{10,11,17}$ which undergo normal transition are illustrated in Figure 9. Contrary to the sytems treated in this study, i.e., ones which undergo inverse transition, the plots for each of these systems is linear at least in the range of temperature indicated, and yields a temperature-independent $\Delta H$. These results suggest that the assumption of constant $\Delta H$, often made in the curve-fitting procedure for evaluating $\sigma$ and $\Delta H$, should be applied with care, especially for transition curves of the inverse type.

\section{DISCUSSION}

\section{Application of Methods $I$ and II}

Vapor pressures above EDC-DCA mixtures are plotted as a function of $1-x_{\mathrm{D}}$ in Figure 10. Here $x_{\mathrm{D}}$ stands for the mole fraction of dimerized DCA. The data points at each temperature follow a straight line quite accurately. Since the vapor pressure of pure DCA was found to be as small as $0.8 \mathrm{mmHg}$ at $40^{\circ} \mathrm{C}$, the highest temperature studied, DCA may be regarded as non-volatile. Therefore, the results of

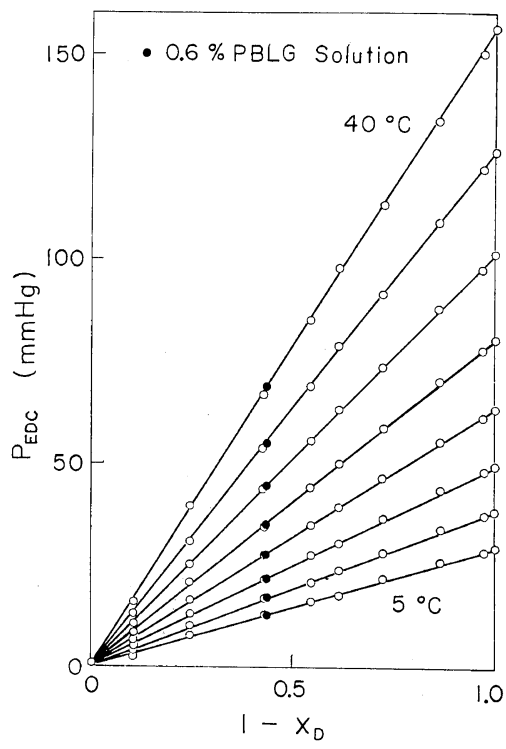

Figure 10. Composition dependence of vapor pressure of EDC, $P_{\mathrm{EDC}}$, for mixtures of DCA and $\mathrm{EDC}$ at $40,35,30,25,20,15,10$, and $5^{\circ} \mathrm{C}$ from top to bottom. $x_{\mathrm{D}}$ denotes the mole fraction of DCA calculated by assuming DCA to exist as dimer.

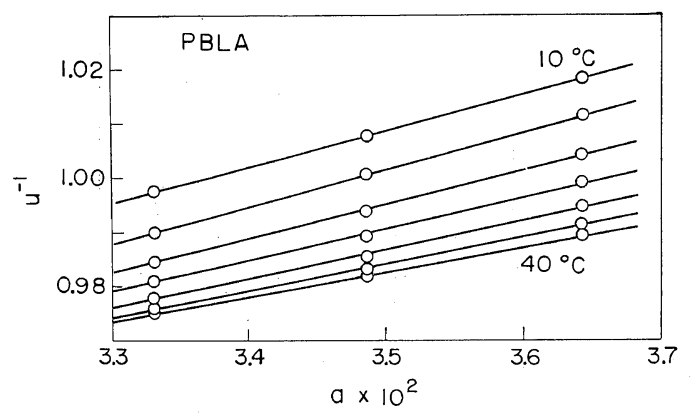

Figure 11. Plots of $u^{-1} v s$. $a$ for PBLA in DCAEDC mixtures at $10,15,20,25,30,35$, and $40^{\circ} \mathrm{C}$ from top to bottom.

Figure 10 indicate that, at least within the range of temperature examined, the system of EDC and dimerized DCA behaves ideally over the entire range of composition. From this finding $a$ was set equal to $x_{\mathrm{D}}$ and $\Delta H_{\mathrm{m}}$ assumed to be zero.

With data for PBLA in DCA-EDC mixtures, the linearity between $u^{-1}$ and $a$ is tested in Figure 11. Although only three points are availabe for each temperature, one may consider that the ex- 


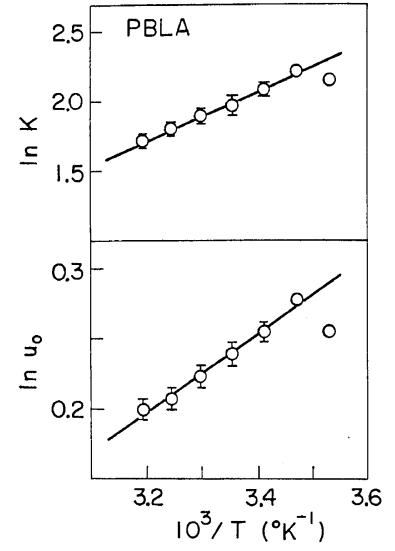

Figure 12. Plots of $\ln K v s .1 / T$ and of $\ln u_{0} v s$. $1 / T$ for PBLA in DCA-EDC mixtures.

pected relationship is well obeyed by experiment. In Figure 12, the values of $\ln K$ and $\ln u_{0}$ derived from the straight lines in Figure 11 are plotted against $1 / T$. The values of $\Delta H_{0}$ and $\Delta H_{\mathrm{a}}$ computed from the slopes of the respective lines are recorded in Table II. Their precision is only moderate.

The data for PBLG in DCA-EDC mixtures were analyzed by Method II. Figure 13 depicts a plot of $u^{-1}$ vs. $a$ at $25^{\circ} \mathrm{C}$ chosen as the reference temperature, $T_{*}$. In this case, too, the data points are seen to follow a straight line as predicted by eq 1 . The intercept of the indicated line at $a=0$ gives 1.69 for $\left(u_{0}\right)_{*}$. Figure 13 also shows $\Delta H_{\mathrm{c}}$ plotted against $\left(1 / T_{\mathrm{c}}-1 / T_{*}\right)$. The values of $P$ and $Q$ estimated from the indicated straight line, together with $\left(u_{0}\right)_{*}=1.69$ and $\Delta H_{\mathrm{m}}=0$ (see above), were used to compute $\Delta H_{0}$ and $\Delta H_{\mathrm{a}}$. The results are reorded in Table II, wherein the values of $T_{\mathrm{c}}{ }^{0}$ calculated from the condition $\ln u_{0}=0$ are also indicated. These $T_{\mathrm{c}}{ }^{0}$ values suggest that both PBLG and PBLA in pure EDC undergo a normal transition in some regions not far above room temperature.

Table II also contains values of $u_{0}$ and $K$ at
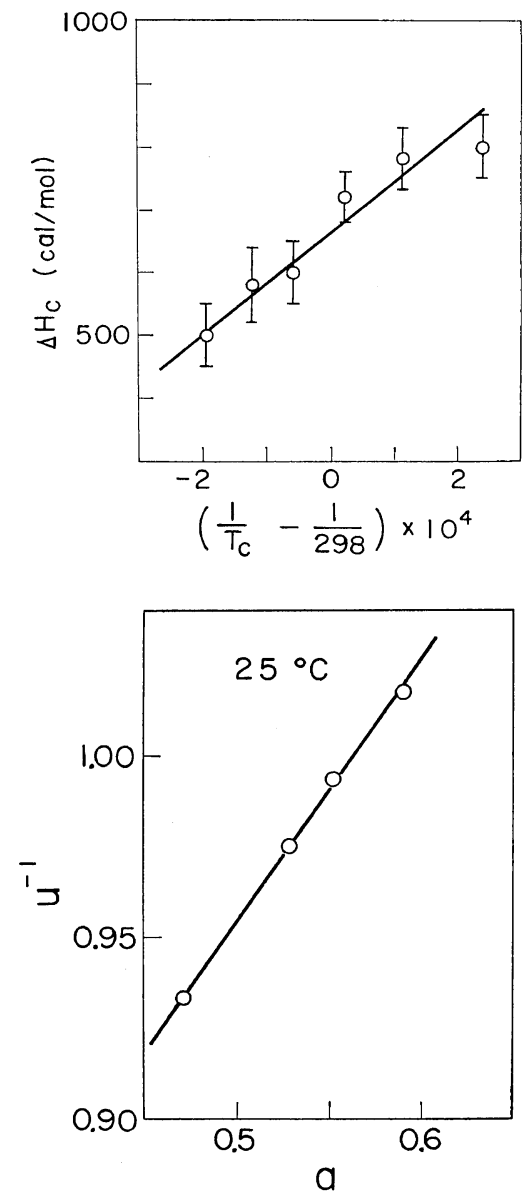

Figure 13. Plots of $\Delta H_{\mathrm{c}} v s .\left(1 / T-1 / T_{*}\right)$ and of $u^{-1}$ vs. $a$ for PBLG in DCA-EDC mixtures. The reference temperature $T_{*}$ is taken to be $298^{\circ} \mathrm{K}$.

$25^{\circ} \mathrm{C}$ and those of the ratio $K a /(1+K a)$ at $25^{\circ} \mathrm{C}$ and appropriate $a$ values for the two systems studied. This ratio indicates what fraction of coiled units is solvated with dimerized DCA at given conditions of temperature and solvent composition. Taken literally, the figures given in Table II indicate that, in either system, a considerable number of peptide units are in the

Table II. Thermodynamic parameters associated with helix-coil transitions of PBLG and PBLA in DCA-EDC mixtures

\begin{tabular}{ccccccc}
\hline Polypeptide & $\begin{array}{c}\Delta H_{0}, \\
\mathrm{cal} / \mathrm{mol}\end{array}$ & $\begin{array}{c}\Delta H_{\mathrm{a}}, \\
\mathrm{cal} / \mathrm{mol}\end{array}$ & $T_{\mathrm{c}}{ }^{0},{ }^{\circ} \mathrm{C}$ & $u_{0}$ & $K{ }^{2}{ }^{\circ}{ }^{\circ} \mathrm{C}$ & $K a /(1+K a)$ \\
\hline PBLA & $-560 \pm 60$ & $-3300 \pm 400$ & $140 \pm 20$ & 1.27 & 7.2 & $0.20(7 \%$ DCA $)$ \\
PBLG & $-800 \pm 300$ & $-3500 \pm 900$ & $210 \pm 80$ & 1.69 & 1.2 & $0.42(75 \%$ DCA $)$ \\
\hline
\end{tabular}


unsolvated state at the conditions specified. In this connection, it is noted that, by analyzing Yang's data ${ }^{18}$ for PBLG in DCA-EDC mixtures, Bixon and Lifson ${ }^{6}$ had arrived at a similar finding. The larger $K$ value implies a stronger interaction between dimerized DCA and peptide unit. It is interesting to observe that the two polypeptides studied have significantly different $K$ values (compared at the same temperature), despite the fact that their side chains differ only by a single methylene group.

The values obtained for $\Delta H_{\mathrm{a}}$ are negative and of the order of magnitude, expected for a hydrogen bond. ${ }^{7}$ They are consistent with the authors' postulated mechanism for the binding of dimerized DCA and peptide unit, because, in this binding, one extra hydrogen bond has to be produced. If a dimerized DCA were to bind independently with each of CO and $\mathrm{NH}$ groups, one should have obtained a much smaller value for $\Delta H_{\mathrm{a}}$, because, in this case, no change occurs in the number of hydrogen bonds before and after the reaction.

Though negative as one should expect, the values obtained for $\Delta H_{0}$ are much lower than those predicted for intramolecular hydrogen bonding of $\mathrm{CO}$ and $\mathrm{NH}$ groups of peptide units. The corresponding entropy changes per rotating bond are calculated to be $-0.7 \mathrm{cal} \mathrm{deg}^{-1}$ for PBLA and $-0.8 \mathrm{cal} \mathrm{deg}^{-1}$ for PBLG: note that the entropy of fusion for an ordinary crystalline polymer is about $2 \mathrm{cal} \mathrm{deg}^{-1}$ per bond. ${ }^{19}$ The rather unexpected results for $\Delta H_{0}$ imply that the formation of a helical structure should be accompanied by certain concomitant processes which either make the helical state of the polypeptide molecule less favorable enthalpically or restrict its conformations in the randomly coil state.

\section{Solvent Effects on $\Delta H$}

Thermally induced helix-coil transitions of polypeptide molecules in dilute solution are classified into two types, normal and inverse, depending on the direction in which the conformational change from helix to coil occurs as the temperature is raised. Thermodynamically, the normal transition appears in temperature regions in which both $\Delta H$ and $\Delta S$ are negative. Here $\Delta S$ is the trrnsition entropy corresponding to the transition enthalpy $\Delta H$. On the other hand, the inverse transition is characterized by positive $\Delta H$ and, $\Delta S$. One of the fundamental problems in the study of helix-coil transition processes in polypeptide solutions is to elucidate which physical factors are responsible for the signs and magnitudes of $\Delta H$ and $\Delta S$.

Equation 6 indicates that the sign of $\Delta H$ is determined by the relative values of the first and second terms on its right-hand side. Since, in general, both $\Delta H_{0}$ and $\Delta H_{\mathrm{a}}-\Delta H_{\mathrm{m}}$ are supposed to be negative, it follows that in order for $\Delta H$ to be positive, the solvent must contain an appropriate amount of an active solvent whose equilibrium constant $K$ for the binding with the peptide unit is moderately large. This prediction conforms to the fact that inverse transitions have so far been observed in mixed solvents which contained a strong acid such as DCA. $^{1,2}$ Another important prediction from eq 6 is that when $\Delta H$ is positive, i.e., when one observes an inverse transition, $\Delta H$ at a fixed solvent composition (or more precisely, at a fixed $a$ value) should decrease with increasing temperature, since $K$ is a decreasing function of temperature. In other words, a plot of $\ln u$ against $1 / T$ at a fixed $a$ should follow a curve which is convex upward. The author's experimental results presented above confirm this prediction.

At a certain temperature, the second term of eq 6 will decrease to the magnitude which is exactly cancelled by the first term, and beyond that temperature, $\Delta H$ becomes negative. Such a change in sign of $\Delta H$ should give rise to a maximum in the helical content $v s$. temperature curve. In fact, it has been confirmed by the authors that the broad maximum observed in Figure 2 corresponded to a particular set of $T$ and $a$ for which $\Delta H$ vanished.

The above argument is restrictive, because eq 6 is based on the particular reaction mechanism assumed for the binding of active solvent and peptide unit. However, its conclusions are essentially similar to those predicted by Peller $^{4}$ on the assumption of a different binding mechanism. The point to notice here is that, in the present investigation, this type of approach to the effect of the active solvent on $\Delta H$ has been brought 
to a quantitative comparison with experimental evidence and its soundness has been demonstrated.

\section{Solvent Effects on $\sigma$}

The values of $\sigma$ obtained for either PBLG or PBLA were essentially independent of temperature and solvent composition. Since $\sigma_{0}$ is supposed to be constant in a relatively narrow range of temperature in which a helix-coil transition is observed, this finding appears to contradict the prediction of eq 2 . Rather it might be taken to imply that the $\sigma$ values obtained actually represent $\sigma_{0}$. However, this idea is not readily acceptable, because it follows from eq 2 that one could obtain $\sigma$ values which were costant within a certain limits of precision if the factor $(1+K a)^{2}$ scarecely varied with temperature and activity $a$ in the main parts of helix-coil transition curves. In fact, numerical computations with the authors' actual $K$ values showed for both PBLG and PBLA that the variation of $(1+K a)^{2}$ with $T$ and $a$ in such regions was comparable to the scatter of the $\sigma$ values estimated. The average values of $\sigma_{0}$ computed from eq 2 with the known values of $\sigma, K$, and $a$ are given in the fifth column of Table III. There is a considerable difference between the $\sigma_{0}$ values of PBLG and PBLA. Differences are also seen to exist in $\Delta H_{0}$ and $T_{\mathrm{c}}{ }^{0}$ (see Table II). Note that all these values still refer to the specific "inert" solvent chosen, EDC. It will be of interest to investigate whether they are really characteristic of the respective polypeptides.

Finally, in the fourth column of Table III, the $\sigma$ values for the systems so far studied in this laboratory are enumerated. As has been the case with the two systems treated in this paper, all these values of $\sigma$ were essentially in- dependent of solvent condition in the ranges investigated. However, the variation observed among the indicated systems is real, exceeding the scatter of the values obtained for each system. It should be noted that the $\sigma$ 's associated with normal transition are consistently higher than those with inverse transition. The data of Gaskin and $\mathrm{Yang}^{21}$ for a series of polydiamino acids in $\mathrm{DCA}-\mathrm{CHCl}_{3}$ mixtures show a distinct parallelism to this fact: $\operatorname{poly}(\delta$ - $N$-carbobenzoxyL-ornithine) $[\Delta H=490 \mathrm{cal} / \mathrm{mol}$ (inverse transition), $\left.\sigma=0.19 \times 10^{-4}\right]$, poly $(\gamma-N$-carbobenzoxy-L- $\alpha, \gamma$-diaminobutyrate) $[\Delta H=-180 \mathrm{cal} / \mathrm{mol}$ (normal transition), $\left.\sigma=0.92 \times 10^{-4}\right]$, and poly( $\varepsilon$-carbobenzoxyL-lysine) $[\Delta H=213 \mathrm{cal} / \mathrm{mol}$ (inverse transition), $\left.\sigma=0.12 \times 10^{-4}\right] .^{22}$ Gaskin and Yang $^{21}$ have concluded from these results that $\sigma$ depends on side chain structure as well as solvent. Their experimental observations are compatible with eq 2 in this paper which predicts that a positive $\Delta H$ is associated with a larger $K a$ value, so that one may obtain relatively low values of $\sigma$ from inverse transition curves, and that the reverse may be the case with normal transition curves.

Equations 1 and 2 are subject to the conditions specified at the beginning of the present development. Different equations have to be used to corrrect observed $\Delta H$ and $\sigma$ for the solvent effect if the active solvent in a given system reacts independently with $\mathrm{CO}$ and $\mathrm{NH}$ groups of peptide units. In this independent-interaction model, as has been assumed by previous authors, ${ }^{4,6,23} K^{\prime}$ may be taken roughly equal to $K$ (Appendix). Then it follows from eq A-5 that $\sigma \cong \sigma_{0}$, which implies that the values of $\sigma$ for such systems may not be affected by the solvent binding. This is probably the case with transitions in aqueous media, conforming to the prediction by $\mathrm{Go}$, et $a l{ }^{23}$

Table III. Comparison of $\sigma$ values for different systems

\begin{tabular}{clcccc}
\hline Polypeptide & \multicolumn{1}{c}{ Solvent } & Type of transition & $\sqrt{\sigma} \times 10^{2}$ & $\sqrt{\sigma_{0}} \times 10^{2}$ & Reference \\
\hline PBLA & DCA-EDC & Inverse & $1.0_{4}$ & 1.7 & This work \\
& DCA-CHCl & Inverse & 0.77 & $(1.5)^{\mathrm{a}}$ & 11 \\
& $m$-Cresol & Normal & $1.2_{6}$ & - & 11 \\
PBLG & DCA-EDC & Inverse & 0.95 & 2.8 & This work \\
& DCA-cyclohexanol & Inverse & $0.9_{2}$ & - & 20 \\
PHPG $^{\mathrm{b}}$ & Water-methanol & Normal & $1.7_{9}$ & - & 10 \\
\hline
\end{tabular}

a Rough estimate from limited data. ${ }^{13}$ b $\operatorname{Poly}\left(N^{5}\right.$-(3-hydroxypropyl)-L-glutamine). 


\section{APPENDIX}

The conformation of a polypeptides chain can be expressed by an array of digits, 0 and 1 , which specify whether the state of each peptide unit is randomly coiled or helical. According to Nagai, ${ }^{8}$ the average conformation of a polypeptide molecule forming an $\alpha$-helix can be determined by assigning a statistical weight to the central peptide unit in each of the seven joint conformations of three consecutive units shown in the first column of Table IV. Nagai has given a general form of the statistical weight matrix to calculate various average quantities as functions of its eigenvalues. The second column of Table IV shows his representation of the statistical weights, with unity taken for the central unit in the 000 conformation. The characteristic equation associated with this statistical weight matrix is

$$
\lambda^{3}(\lambda-\sigma)(\lambda-1)-\sigma \alpha_{1} \alpha_{2}\left[\gamma_{1} \gamma_{2}+\gamma_{3}(\lambda-1)\right]=0
$$

Based on a physical reason, Nagai has simplified this equation to a form which, in the authors' present notation, may be written

$$
\lambda^{2}(\lambda-1)\left(\lambda-u_{0}\right)=\sigma_{0} u_{0}
$$

This is equivalent to the characteristic equation of Zimm and Bragg. ${ }^{9}$ The statistical weights simplified by Nagai are given in the third column of Table IV.

Although eq A-1 and A-2 have been derived without explicit consideration of the binding of an active solvent, it is possible to incorporate this effect in them by use of a procedure similar to that described by Peller ${ }^{4}$ and also by Bixon and Lifson. ${ }^{6}$ Those authors proposed multiplying the statistical weight of a peptide unit by a factor of $(1+K a)$ if the unit considered contains a site which is accessible to hydrogen bonding with the active solvent. Here $K$ is the equilibrium constant for the hydrogen bonding, and $a$ is the activity of the active solvent.

In the model used by Bixon and Lifson, ${ }^{6}$ in which each of $\mathrm{CO}$ and $\mathrm{NH}$ groups of peptide units is assumed to react independently with the active solvent, the relevant factor to be asigned to the central units in the seven joint conformations become as shown in the fifth column of Table IV. For example, the central unit in the 101 conformation has no free active site, so that its statistical weight should be multiplied by unity. Obviously, the factor $\left(1+K_{\mathrm{CO}} a\right)(1+$ $K_{\mathrm{NH}} a$ ) should be assigned to the central unit in the 000 conformation. For the central unit either in the 011 conformation or in the 110 conformation, one needs a deliberate treatment. This unit, i.e., the terminal unit of a helical section, and the two helical units which follow have either a free $\mathrm{NH}$ or a free $\mathrm{CO}$ group in each. As long as one assigns the value of $u_{0}$ to the statistical weight of any unit inside a helical section, it becomes necessary to treat it as if the terminal helical unit in question had three active $\mathrm{NH}$ or $\mathrm{CO}$ groups and the following two helical units underwent no binding of the active solvent. Based on this consideration, one may assign the factor $\left(1+K_{\mathrm{NH}}{ }^{\prime} a\right)^{3}$ or $\left(1+K_{\mathrm{CO}}{ }^{\prime} a\right)^{3}$ to the central unit in either the conformation 011 or the conformation 110. Here the prime indicates that the active $\mathrm{NH}$ or $\mathrm{CO}$ group is in a helical unit.

Table IV. Effects of solvent binding on statistical weights of peptide units

\begin{tabular}{ccccc}
\hline \multirow{2}{*}{ Conformation } & \multicolumn{4}{c}{ Statistical weights of central units } \\
\cline { 2 - 5 } & $\begin{array}{c}\text { Nagai's } \\
\text { original }\end{array}$ & $\begin{array}{c}\text { Nagai's } \\
\text { simplification }\end{array}$ & $\begin{array}{c}\text { Present } \\
\text { model }\end{array}$ & $\begin{array}{c}\text { Independent-interaction } \\
\text { model }^{\mathrm{a}}\end{array}$ \\
\hline 111 & $\sigma$ & $u_{0}$ & $u_{0}$ & $u_{0}$ \\
101 & $\gamma_{3}$ & 1 & 1 & 1 \\
011 & $\alpha_{2}$ & $\sqrt{\sigma_{0}}$ & $\sqrt{\sigma_{0}}$ & $\left(1+K_{\left.\mathrm{CO}^{\prime} a\right)^{3}} \sqrt{\sigma_{0}}\right.$ \\
001 & $\gamma_{1}$ & 1 & 1 & $\left(1+K_{\mathrm{CO} a)}\right.$ \\
110 & $\alpha_{1}$ & $\sqrt{\sigma_{0}}$ & $\sqrt{\sigma_{0}}$ & $\left(1+K_{\mathrm{NH}}^{\prime} a\right)^{3} \sqrt{\sigma_{0}}$ \\
100 & $\gamma_{2}$ & 1 & 1 & $\left(1+K_{\mathrm{NH}} a\right)$ \\
000 & 1 & 1 & $1+K a$ & $\left(1+K_{\mathrm{CO} a)\left(1+K_{\mathrm{NH}} a\right)}\right.$ \\
\hline
\end{tabular}

a Gō, et al., ${ }^{23}$ adopted essentially equivalent but somewhat different definitions for statistical weights. 
After normalization with respect to the central unit in the 000 conformation, the statistical weights shown in the last column of Table IV are substituted into eq A-1 to give

$$
\lambda^{2}(\lambda-1)(\lambda-u)=\sigma u
$$

where

$$
\begin{aligned}
u & =u_{0} /\left(1+K_{\mathrm{CO}} a\right)\left(1+K_{\mathrm{NH}} a\right) \\
\sqrt{\sigma} & =\sqrt{\sigma_{0}}\left[\frac{\left(1+K_{\mathrm{CO}}{ }^{\prime} a\right)\left(1+K_{\mathrm{NH}}{ }^{\prime} a\right)}{\left(1+K_{\mathrm{CO}} a\right)\left(1+K_{\mathrm{NH}} a\right)}\right]^{3 / 2}
\end{aligned}
$$

Equation A-3 is identical in form to eq A-2, except that $u_{0}$ and $\sigma_{0}$ are here replaced by $u$ and $\sigma$, respectively. Thus one finds that when the solvation mechanism assumed by Bixon and Lifson is obeyed, the parameters $u$ and $\sigma$ evaluated by applying eq A-3 to experimental data can be equated to the quantities defined by eq $\mathrm{A}-4$ and A-5. In this case, if it happens that $K_{\mathrm{CO}}=K_{\mathrm{CO}}{ }^{\prime}$ and $K_{\mathrm{NH}}=K_{\mathrm{NH}}{ }^{\prime}$, (i.e., if for either active site the equilibrium constant is not affected by whether the site is in the helical unit or in the coiled unit), it follows from eq A-5 that $\sigma=\sigma_{0}$, i.e., $\sigma$ undergoes no effect by the solvent binding. This is the conclusion of the previous authors ${ }^{6,23}$ who assumed the independent interaction.

For the model assumed in the present development, in which a dimerized DCA reacts with a pair of $\mathrm{CO}$ and $\mathrm{NH}$ groups, one must assign different factors to the central units in the seven joint conformations. In this model, central units other than that in the 000 conformation can take part in the reaction with the active solvent. Thus the statistical weights of the seven central units become as shown in the fourth column of Table IV. Here $K$ is the equilibrium constant for the reaction considered, and as before, $a$, denotes the activity of the dimerized DCA. Substitution of these statistical weights into eq A-1 yields

$$
\lambda^{2}(\lambda-1)(\lambda-u)=\sigma u\left[1+\frac{(\lambda-1)}{\lambda} K a\right]
$$

with

$$
\begin{gathered}
u=u_{0} /(1+K a) \\
\sqrt{\sigma}=\sqrt{\sigma_{0}} /(1+K a)^{2}
\end{gathered}
$$

For $\sqrt{\sigma} \ll 1$, the largest root $\lambda$ of eq A- 6 can be expressed by

$$
\lambda=1+0(\sqrt{\sigma})
$$

Hence eq A-6 may be written in the form:

$$
\lambda^{2}(\lambda-1)(\lambda-u)=\sigma u[1+0(\sqrt{\sigma})]
$$

This indicates that, using the approximation that the term multiplied by $\sqrt{\sigma}$ may be ignored in comparison with unity, the characteristic equation associated with the authors' reaction model becomes the same in form as that for the independent-interaction model of Bixon and and Lifson ${ }^{6}$ and of Peller. ${ }^{4}$ Therefore, when the authors's model is applicable, analysis of experimental data for helical content yields (though not exactly) the values of $u$ and $\sqrt{\sigma}$ which can be equated to the right-hand sides of eq A-7 and A-8, respectively. Finally, it is noted that, while $u$ represents the statistical weight of the central unit in the 111 conformation relative to that of the coiled unit in the 000 conformation, the quantity $\sqrt{\sigma}$ does not necessarily have physical meaning as the corresponding statistical weight of the terminal helical unit of a helical section.

\section{REFERENCES}

1. G. D. Fasman, in "Poly- $\alpha$-Amino Acids," G. D. Fasman, Ed., Marcel Dekker, Inc., New York, N.Y., 1967, Chapter 11.

2. P. Doty, and J. T. Yang, J. Amer. Chem. Soc., 78, 498 (1956).

3. J. A. Schellman, Compt. rend. trav. lab. Carlsberg, Ser. chim., 29, 230 (1955); J. Phys. Chem., 62, 1485 (1958).

4. L. Peller, J. Phys. Chem., 63, 1194, 1199 (1959).

5. J. H. Gibbs and E. A. DiMarzio, J. Chem. Phys., 30, 271 (1959).

6. M. Bixon and S. Lifson, Biopolymers, 4, 815 (1966).

7. G.C. Pimentel and A. L. McClellan, "The Hydrogen Bond," Freemen \& Co., San Francisco and London, 1959.

8. K. Nagai, J. Chem. Phys., 34, 887 (1961).

9. B. H. Zimm and J. K. Bragg, J. Chem. Phys., 31, 526 (1959).

10. K. Okita, A. Teramoto, and H. Fujita, Biopolymers, 9, 717 (1970).

11. Y. Hayashi, A. Teramoto, K. Kawahara, and H. Fujita, Biopolymers, 8, 403 (1969).

12. T. Norisuye, M. Matsuoka, A. Teramoto, and H. Fujita, Polymer J., 1, 691 (1970).

13. A. Teramoto, unpublished data. 
14. “Organic Solvents," A. Weissberger, E. S. Proskauer, J. A. Riddick, and E. E. Toops, Jr., Ed., Interscience Publishers, Inc., New York, N.Y., 1955 , p 196.

15. A. Nakajima and T. Hayashi, Bull. Inst. Chem. Res., Kyoto Univ., 46, 62 (1968).

16. A. Teramoto and T. Norisuye, Biopolymers, in press.

17. R. T. Ingwall, H. A. Scheraga, N. Lotan, A. Berger, and E. Katchalsky, Biopolymers, 6, 331 (1968).
18. J. T. Yang, Tetrahedron, 13, 143 (1961).

19. P. J. Flory, "Principles of Polymer Chemistry," Cornell Univ. Press, Ithaca, N.Y., 1953.

20. T. Norisuye, A. Teramoto, and H. Fujita, to be published in Polymer Jornal.

21. F. Gaskin and J. T. Yang, Biopolymers, 10, 631 (1971).

22. F. E. Karasz and J. M. O'Reilly, Biopolymers, 3, 241 (1965).

23. M. Gō, N. Gō, and H. A. Scheraga, J. Chem. Phys., 52, 2060 (1970); ibid., 54, 4489 (1971). 